\title{
FUNCTIONAL ANALYSIS OF SMALL UBIQUITIN-MODIFIER (SUMO) IN ORANGE-SPOTTED GROUPER (EPINEPHELUS COIOIDES)
}

\author{
Young-Mao Chen ${ }^{1,3,4}$, Yu-Ting Kao ${ }^{1,3}$, Tzong-Yueh Chen ${ }^{1,2,3,4}$ § \\ ${ }^{1}$ Institute of Biotechnology, National Cheng Kung University, Tainan 70101, Taiwan \\ ${ }^{2}$ Department of Biotechnology and Bioindustry Sciences, National Cheng Kung University, Tainan \\ 70101, Taiwan \\ ${ }^{3}$ Translational Center for Marine Biotechnology, National Cheng Kung University, Tainan 70101, \\ Taiwan \\ ${ }^{4}$ Agriculture Biotechnology Research Center, National Cheng Kung University, Tainan 70101, Taiwan
}

\begin{abstract}
Small ubiquitin-like modifiers (SUMOs), structurally similar to ubiquitin, ligated to lysine residues within sumoylation target proteins. Sumoylation and ubiquitination exhibit similar biological processes for post-translational modification regulation, apoptosis and protein stability which was participated in a number of cellular processes such as nuclear transport, transcriptional regulation, apoptosis and protein stability. In the present study, the cDNA of orange-spotted grouper SUMO, terms osgSUMO, was cloned by the combination of homology cloning and rapid amplification of cDNA ends polymerase chain reaction (RACE-PCR) approaches. The SUMO1 and SUMO2-yellow fluorescent protein (YFP) were distributed in the cytoplasm. Our results show that SUMO1 was capable of decreasing coat protein (CP) of nucleus localization ratio, not SUMO2. Thus, we demonstrate here that SUMO1 was able to interact with CP by using analysis of fluorescence resonance energy transfer (FRET). Taken together, grouper SUMO can partially influence on localization of CP.
\end{abstract}

\section{KEYWORDS}

Small ubiquitin-like modifiers (SUMOs), Epinephelus coioides, nodavirus, sumoylation, fluorescence resonance energy transfer (FRET)

§Corresponding author. Tel.: +886-6-2757575\#65622-610; Fax: +886-6-2766505

E-mail address: ibcty@mail.ncku.edu.tw 